\title{
Notfalldienst 2002
}

\author{
C. Eugster
}

Die sanften und harmonisch aufeinander abgestimmten, aber unbarmherzig fortgesetzt sich wiederholenden Klingeltöne meines Telefons entreissen mich unvermittelt aus schönen Träumen und kurzem, allerdings tiefem Schlaf. Ich schalte das warme, durch einen hellen Stoffschirm gedämpfte Licht des Nachttischlämpchens ein, schaue hastig auf meine Uhr, sie zeigt die Zeit von halb vier Uhr morgens an. Am Telefon meldet sich die Stimme einer wohl jüngeren Frau, geschäftsmässig und indifferent klingt sie.

«Herr Doktor, ich habe gehört, dass Sie Notfalldienst haben, ist dies richtig?»

«a, dies trifft zu», bestätige ich mit leiser, noch verschlafener Stimme. «Ja, ich habe städtischen Notfalldienst.»

«Hören Sie, seit genau Mitternacht quälen mich Schmerzen im Unterbauch sowie ständiger Harndrang, zudem brennt das Wasserlösen stark und es verursacht weitere Schmerzen, ich habe auch etwas Blut im Wasser gesehen. Ich möchte, dass Sie etwas dagegen tun und mir etwas geben, wann kann ich zu Ihnen kommen?» Nun, was soll ich der jüngeren Frau antworten, soll ich ihr zu einer Eigenmedikation raten und ihr empfehlen, viel Flüssigkeit zu trinken sowie einen warmen Wickel zu machen oder soll ich besser ihrem Wunsch Folge leisten und sie in der Praxis behandeln?

Die Frauenstimme kommt meiner Antwort zuvor: «Wissen Sie, ich habe bereits sehr viel Tee getrunken und Medikamente zur Behandlung habe ich nicht bei mir. Eigenartigerweise sind genau gleiche Beschwerden vor Monatsfrist bereits aufgetreten, ebenfalls um Mitternacht, auch damals brauchte ich einen Notfallarzt. Können Sie mir vielleicht beantworten, warum dies jetzt erneut passiert ist? Und ich wäre schon froh, wenn Sie dies abklären könnten.»

Die Beantwortung ihrer Frage am Telefon erspare ich mir, und im Wissen darum, dass eine Zystitis im Trigonum-Bereich sehr unangenehm sein kann, offeriere ich der Anruferin einen Termin um vier Uhr morgens in der Praxis, also in einer halben Stunde. Hätte ich ihr geantwortet, sie solle Tee trinken, ein Schmerzmittel einnehmen und am kommenden Vormittag ihren Arzt aufsuchen, würden wir beide nicht zufrieden sein, sie nicht aufgrund einer schlaflosen Restnacht der lästigen Symptome wegen und ich nicht aufgrund einer schlaflosen Restnacht der infolge meiner ihre Beschwerden ignorierenden Antwort aufkommenden Gewissensbisse wegen.

«Also komme ich in einer halben Stunde in Ihre Praxis», flötet es durch den Telefonhörer. «Können Sie mir noch genau erklären, wie ich zu ihr gelange, und hat es Parkplätze vor dem Haus?»

Geduld braucht ein Arzt, auch während der Nacht. Schlaftrunken und etwas wehmütig auf die noch warme Bettdecke und das kopfformgerecht zusammengedrückte Kopfkissen schauend, ziehe ich mich an und mache mich für die kurze Fahrt zur Praxis zurecht. Gedanken zum bisherigen Verlauf des Notfalldienstes kommen auf. Nein, wirklich streng ist der Dienst an diesem Freitag, dem 25. Oktober 2002, bisher noch nicht gewesen. Während des Tages verzeichnete ich vier oder fünf Konsultationen von Personen, die mich notfallmässig in Abwesenheit oder wegen Überlastung ihres Hausarztes aufgesucht hatten, Rückenschmerzen, grippale Infekte, Kopfschmerzen und Durchfall waren die Gründe hiefür gewesen, medizinisch gesehen leichte Fälle, daneben gab es einige Telefone, unvermeidliche Telefone verunsicherter Leute, oh wie liebe ich die Handys, die während des Gesprächs aufgrund der Durchfahrt eines Tunnels oder auf kurvenreicher bergiger Strecke, beides gibt es in der Stadt, in der ich arbeite, sowie in deren Umgebung in reichlichem Masse, plötzlich abstellen und einen dann voraussehbar und vorausberechenbar eine Minute später erneut zum Telefonhörer befehlen, «Äh, ich hätte noch gerne Ihre Antwort auf meine kurze Frage gehört», oder «Können Sie mir noch einmal sagen, was Sie mir vorhin erklärt haben, ich musste mich leider auf den Verkehr konzentrieren», ja und Handys sind neben ihrer erwiesenen Nützlichkeit für den Notfalldienst und andere Tätigkeiten durchaus auch segensreich für die Netzbetreiber, vor allem in Voralpen- und Bergregionen, wo man für ein Gespräch mit dem Schlusswort Adieu oft zwei oder drei Anrufe benötigt.

Die Sprechstunde tagsüber hatte ihren gewohnten Lauf genommen, natürlich gab es auch zahlreiche Notfallkonsultationen von regulär von mir betreuten Patienten, aber dies gehört zum normalen Praxisalltag. Am frühen Abend sah ich im Rahmen des städtischen Notfalldienstes noch einen Schüler mit einer lakunären Angina, einer bei Ärzten und Ärztinnen in der Praxis und spe- 
ziell im Notfalldienst beliebten Diagnose, da sie sich leicht und rasch stellen und sich in der Regel gut behandeln lässt, und die Mehrzahl der Betroffenen mit der erbrachten ärztlichen Leistung zufrieden ist, ausserdem einen Ferienrückkehrer aus dem Kosovo mit während des Rückflugs aufgetretenen starken Kreuzschmerzen, wobei auch der ohne Vorankündigung mitgebrachte alte Vater über Rückenbeschwerden klagte und behandelt werden wollte, aber seine Schmerzen bestünden schon seit Monaten oder Jahren, und dann gab es überdies den Anruf eines jungen Mannes, der über chronische Kopfschmerzen berichtete, tagsüber aber nie zum Arzt gehen könne, da er immer arbeiten müsse, eine Abklärung sei aber sicher nötig, dies wisse er schon, und ihm konnte ich mit einigen von seiner Seite her akzeptierten Ratschlägen und sogar unter Auslassung der gewünschten Abklärungen am Abend helfen, ohne dabei Gewissensbisse wegen möglicherweise unterlassener Hilfeleistung zu verspüren. So war mir in der Folge auch Schlaf vergönnt gewesen, kurzer zwar, aber immerhin welcher.

Die Notfallpatientin kommt mit einer Verspätung von fünfzehn Minuten in meine Praxis, ach wie langsam läuft der Minutenzeiger meiner Armbanduhr beim nächtlichen Warten auf einen Notfall und wie rasch kreist er jeweils während der Arbeit am Tage auf dem Zifferblatt herum! Die Frau ist tatsächlich jüngeren Alters, sie wirkt locker und hat ein gewandtes Auftreten.

«Wissen Sie, Herr Doktor, in der Dunkelheit ist es noch schwieriger als am Tag, sich in der Stadt nicht zu verfahren, aber jetzt habe ich es geschafft, Sie zu finden, ich bin froh darüber.»

Ich untersuche ihren Urin, auffallend hell ist er, natürlich verdünnt ob der zuvor eingenommenen Mengen an Tee, immerhin sind im Schnelltest mehrere Parameter positiv, nicht aber die Nitritprobe, doch das spezifische Gewicht ist aufgrund der Verdünnung niedrig, womit diesem Test in dieser Situation keine diagnostische Aussage zukommt. Fieber hat die Patientin nicht und Flankenschmerzen verneint sie. Ob sie regelmässig Medikamente einnehme, frage ich sie gewissenhaft, und sie antwortet mir, ja doch, sie nehme täglich Medikamente ein, von ihrem Homöopathen erhalte sie Globuli und Tropfen und von ihrem Psychotherapeuten ein Präparat, an dessen Namen sie sich zurzeit allerdings nicht zu erinnern vermöge, aber zugegeben, sie schlucke es nur unregelmässig, so hin und wieder, in ihrem hektischen Beruf als Direktionssekretärin vergesse sie dessen Einnahme sowieso häufig. Ich bespreche die Situation mit ihr, bestätige ihre Annahme einer Blasenentzündung und rate zur geregelten Einnahme eines
Chinolon-Präparates und zur Verwendung eines krampf- und schmerzlösenden Mittels nach Bedarf, hierauf schaffe ich gängige Präparate herbei.

«Das Antibiotika (natürlich ist dies falsch geschrieben, aber ich höre es von der Notfallpatientin und im übrigen immer wieder auch von vielen anderen Leuten so ausgesprochen) ist in Ordnung, Herr Doktor, aber dieses Analgetika (oh meine Ohren) vertrage ich nicht, darauf reagiere ich allergisch, nein, dies geht nicht», vernehme ich, also wird letzteres Präparat zurückgezogen, zum Glück habe ich dessen Verpackung noch nicht mit den nötigen schriftlichen Einnahmeanweisungen versehen und ich empfehle statt seiner das sicher unproblematische Paracetamol in der hierzulande billigsten Form.

«Gut, ich versuche es, gehört habe ich davon allerdings noch nie», höre ich die leicht warnende Stimme meines Gegenübers.

«Es ist tatsächlich unbedenklich», versichere ich ihr, schaue sie an und füge hinzu: «Besser nehmen Sie eine grössere Schachtel des Antibiotikums mit, da ...», doch hier fällt sie mir schroff ins Wort mit dem Hinweis, wonach die kleine Schachtel mit der Hälfte des Inhalts der grösseren durchaus genüge.

«Nein, diese genügt durchaus nicht», entgegne ich leicht gereizt und mit gedehnter Stimme. «Für die Behandlung jetzt reicht zwar die eine Hälfte des Inhalts, aber die andere Hälfte wollen Sie bitte dann gebrauchen, falls sich wieder eine nächtliche Blasenentzündung ankündigen sollte.»

«Gute Idee, Herr Doktor, dann meinen Sie, dass ich vielleicht bei einem weiteren Mal keinen Notfallarzt zu beanspruchen brauche?»

Ich nicke zustimmend, wir haben uns verstanden. Ihre noch einmal gestellte Frage nach der Ursache ihrer Blasenentzündung beantworte ich mit der Erklärung, die mir am wahrscheinlichsten zu sein scheint und stelle fest, dass wir uns erneut verstehen. Im weiteren rate ich ihr, regelmässig prä- und wenn möglich auch postkoital die Harnblase zu entleeren und generell viel zu trinken. Dies habe sie auch schon einmal gelesen, in einer Frauenzeitschrift, erinnert sich die Notfallpatientin und sie bekräftigt, meine Ratschläge nach Möglichkeit zu befolgen, aber leicht gelinge deren Umsetzung durchaus nicht immer. Ergänzend informiere ich sie dahingehend, dass die Wirkung der Medikamente nicht sofort eintrete, es brauche hiefür leider einige Stunden. Mit einem Glas Wasser nimmt die Frau je eine Tablette ein, verlangt nach einem weiteren Glas, eigentlich vorbildlich, und sucht in der Folge noch einmal für längere Zeit die Toilette 
auf. Ich zeige Verständnis, zumal ja immer noch vermehrter Harndrang besteht. Hierauf verabschieden wir uns, ich fahre zurück nach Hause, und der Minutenzeiger meiner Uhr ist seit meinem letzten Blick darauf wieder einmal viel zu schnell im Kreis herum gefahren. Müde sinke ich ins kalt gewordene Bett, auch vorsichtig, um meine liebe Frau nicht ein weiteres Mal zu wecken und entrücke bald wieder in irgendeinen Traum.

Eine knappe Stunde später, um halb sechs Uhr morgens, klingelt das Telefon erneut. Ich schrecke hoch, nehme den Hörer ab und vernehme zu meinem zweiten Schrecken wieder die monoton geschäftsmässig tönende Stimme der Direktionssekretärin.

«Herr Doktor, ist es wirklich normal, dass ich immer noch ständigen Harndrang sowie Brennen beim Wasserlösen spüre?»

«a, dies ist wirklich normal in dieser Situation, haben Sie noch zwei bis drei Stunden Geduld, so wie ich es Ihnen vor Ihrem Weggehen erklärt habe.»

Seufzend lege ich den Telefonhörer wieder auf, ich stelle fest, dass wir uns bei meiner letzten Information in der Praxis durchaus nicht verstanden haben.

Der Morgen kommt mit leisem und seidnem Sonnenschein, allein ich fühle mich müde und zerschlagen, der Schädel brummt, und der Schlaf vorhin ist alles andere als erquickend gewesen. Vermehrte Tagesmüdigkeit wird mich erwarten, bin ich ein Kandidat für das Schlaflabor im nahegelegenen Kantonsspital?

Der Praxisbetrieb des nächsten Praxistages beginnt, heute sind viele Leute eingeschrieben, wie immer an einem Samstagmorgen, trotz meiner Einschränkung der Befindlichkeit können sie nicht ausgeschrieben werden, und im weiteren werden wieder Notfälle kommen, aus meinem Sprengel, so ist es eben, und es wird Notfälle geben, bei denen ich in bezug auf die Definition dieses Begriffes die gleiche Meinung haben werde wie die Betroffenen, aber auch solche, bei denen sich unsere Ansichten durchaus nicht decken, wo wir uns diesbezüglich nicht verstehen werden. Andererseits respektiere ich bei jedem notfallmässig erscheinenden Menschen, dass er derjenige ist, welcher die Beschwerden hat, und nicht ich, der sie lediglich zu verstehen hat.

So ist im Grunde jeder Tag in der Praxis ein Tag mit Notfalldienst, und die nächtlichen Notfälle sind im wesentlichen nicht anders als die täglichen, aber zugegeben, sie belasten Körper und Geist der praktizierenden Ärzte und Ärztinnen ungleich mehr als die letzteren. Doch ich habe einmal ein klares und lautes Ja zu diesem meinem Beruf gesagt und dieses Ja will ich nicht zurückziehen, will es auch nicht leiser und schwächer werden lassen, ich stehe zu ihm.

Übrigens, als Nachlese zu meinem eben absolvierten offiziellen städtischen Notfalldienst erhalte ich um halb neun Uhr vormittags einen weiteren dringenden Anruf der Notfallpatientin der vergangenen Nacht, von einem Handy aus, hoffentlich durchquert sie nicht demnächst einen der zahlreichen Tunnels unserer Stadt.

«Hallo Herr Doktor, haben Sie gut angefangen mit der Arbeit?» Ach, wie praktisch und kommunikationsfördernd ist doch ein Handy! Ärger beginnt in mir hochzusteigen und ich murmle etwas vor mich hin.

«Also, meine Beschwerden haben jetzt deutlich nachgelassen, erfreulicherweise, aber meine nun brennende Frage an Sie ist, wie Sie sich zu einem von mir für heute Nachmittag vorgesehenen Flug stellen, wissen Sie, zu einem Städteflug nach Spanien, nur für ein paar Tage. Gebucht habe ich zwar schon, zusammen mit meinem Partner, aber ich möchte Sie trotzdem um Ihre Meinung fragen.»

Ich überlege kurz, bin ich nicht immer noch leicht verärgert? Dann bejahe ich ihre Frage, schlucke aufkeimende Bedenken hinunter, zumal ihre Frage vorhin doch nichts anderes als eine reine Sicherheitsfrage zur Beruhigung ihres Gewissens oder anders ausgedrückt eine medizinische Absicherungsfrage gewesen ist, und ein Einwand meinerseits zwar nicht zur Stornierung der Flugreise führen, möglicherweise aber doch eine schwere Verunsicherung bei der Frau auslösen würde, rate ihr aber erneut zur wirklich regelmässigen Einnahme des Antibiotikums, äh, ob ich damit wohl das Antibiotika meine, auf die Wiederholung der anderen gemachten Vorschläge verzichte ich, in diesem Punkt hatten wir uns ja verstanden und wünsche ihr einen schönen Aufenthalt in Spanien.

Ich hänge den Hörer auf und widme mich wieder dem mir gegenübersitzenden älteren und schwerhörigen Sprechstundenpatienten, als es an der Türe klopft. Eine Praxisassistentin tritt ein und fordert mich auf, doch schnell zu einem Notfall im Nebenzimmer zu kommen, einer jungen Frau gehe es schlecht, sie erbreche dauernd. Und hinter ihr, die in der geöffneten Türe steht, sehe ich weitere regulär und nicht regulär eingeschriebene Patienten in meine Praxis eintreten. Zwar leuchtet die Sonne draussen sanft und seiden, aber das Licht in meinem Sprechzimmer ist hart und brennend, dergestalt, dass es in meinen Augen und in meiner Kehle weiterbrennt. Doch ich weiss, gegen Mittag wird es wärmer und weicher scheinen, und ich werde mich dann besser fühlen, und morgen wird Sonntag sein. 